\title{
Sparse and Adaptive Sampling in Scanning Electron Microscopy
}

\author{
$\underline{\text { Tim Dahmen }}^{1}$ and Patrick Trampert ${ }^{1,2}$
}

${ }^{1 .}$ Deutsches Forschungszentrum für Künstliche Intelligenz GmbH, Saarbrücken, Germany.

2. Saarland University, Saarbrücken, Germany.

In scanning electron microscopy, a focused electron beam scans over the specimen, such that an image is acquired row-by-row (raster scan). In order to improve image quality beyond the limits dictated by electron statistics, information from other sources than the detector, prior knowledge, must be included in the imaging process. Depending on the nature of this prior knowledge, acquiring the image row-by-row with constant dwell time per pixel is not optimal and other acquisition strategies can result in superior results. In the following, we give an overview of sparse and adaptive scan patterns and corresponding reconstruction algorithms for electron microscopy.

We first consider the possibilities of non-adaptive scan patterns. Assuming prior knowledge in the form of an over-complete dictionary of image patches, several strategies are possible. One can perform a raster scan with a reduced dwell time per pixel, followed by a state-of-the-art Denoising algorithm such as Geometric Analysis Operator Learning (GOAL) Denoising. Alternatively, raster scanning with an increased spot size (i.e. decreased resolution) can be combined with a state-of-theart Super Resolution algorithm. Thirdly, a random sparse scan, where a fixed percentage of randomly selected pixels is visited by the beam, can be combined with state-of-the-art inpainting algorithms such as Exemplar-Based Inpainting, GOAL Inpainting, or Beta Process Factor Analysis (BPFA) Inpainting. An experimental comparison of the three approaches showed superior results for a random sparse scan in combination with BPFA Inpainting, at least for one specific microscope setup and a specimen consisting of mouse brain tissue [1].

A critical factor in this study was the control of the electron beam. Random scanning methods in general are very sensitive to drift. This is the case as the measurement of two pixels that are spatially located very close to each other can be separated by a relatively long period of time. Accumulated drift then leads to strong local distortions. A solution to the problem is to scan the pixels in an order according to their position on a Hilbert curve. This approach enforces a correlation between scan time and position and drastically reduces the overall distance that the beam needs to travel in order to visit all pixels [2].

We now consider adaptive dense scan patterns. Adaptive scan patterns are potentially superior to non-adaptive patterns but must be tailored to one specific form of prior-knowledge. The easiest possible scenario underlies the assumption that the image has a sparse gradient, i.e. the specimen consists of homogeneous areas with sharp boundaries. An adaptive dense scan method for this case is Feature Adaptive Sampling for Scanning Electron Microscopy [3]. In this technique, the sample was scanned twice. The first scan used small pixel-dwell times to generate an initial, noisy image using a low electron dose. This image was analyzed automatically, and an algorithm generated a sparse pattern of regions of the image that required additional sampling. A second scan generated a sparse image of only these regions, but using a highly increased electron dose. By applying a selective low-pass filter and combining both datasets, a single image was generated. The resulting image exhibited a factor of $\approx 3$ better SNR corresponding to a factor of ten lower dwell time compared to uniform raster scanning (Figure 1).

We finally consider adaptive sparse scan patterns. In these patterns, only a subset of pixels is ever measured, but an algorithm makes the decision which pixels should be measured next. The approach improves over the previously presented method by avoiding the relatively costly (in terms of total dwell time) initial scan. Using again the assumption of a sparse image gradient, the sampling is 
initialized with a sparse set of randomly selected pixels. Neighborhood relations between sampling positions are established by building a Voronoi diagram. Additional samples are placed incrementally on the edges of the diagram if the image gradient along the edge exceeds a subdivision criterion (Figure 2).

In conclusion, scanning electron microscopy can be improved by combing measured data with additional, prior knowledge. Sparse and adaptive sampling techniques will play a central role in this development. Key factors are the mathematical representation of the prior knowledge and as precise as possible control over the electron beam. [4]

\section{References:}

[1] P Trampert et al, Ultramicroscopy 191 (2018), p. 11.

[2] F Boughorbel et al, Microsc Microanal 23 (S1) (2017), p. 150.

[3] T Dahmen et al, Scientific Reports 6 (2016), p. 25350.

[4] The authors thank Thermo Fischer Scientific, DFKI GmbH, Saarland University, and INM $\mathrm{GmbH}$ for funding the research.

a)

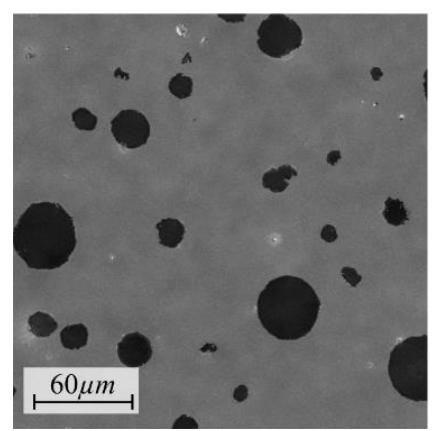

b)

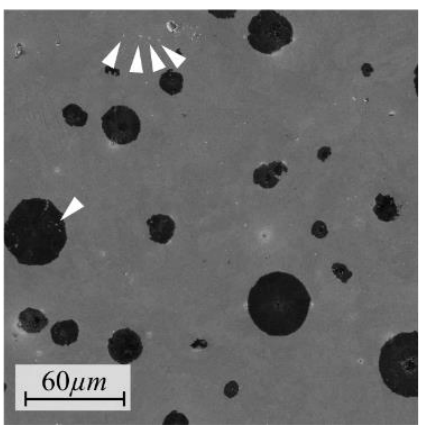

c)

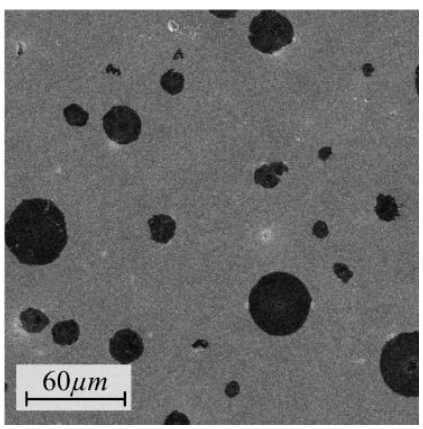

Figure 1. Feature adaptive sampling. a) Our method using an average pixel-dwell time of $3 \mu \mathrm{s}$. b) A uniform pixel-dwell time of $60 \mu$ s (ground truth). c) Uniform sampling at $3 \mu$ s per pixel. d) Uniform pixel-dwell time of $3 \mu$ s per pixel and filtered using an anisotropic diffusion operator.

a)

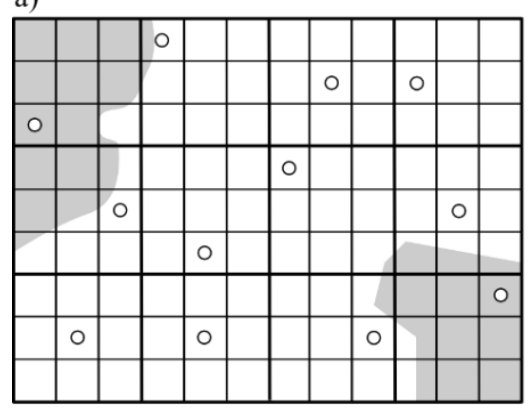

b)

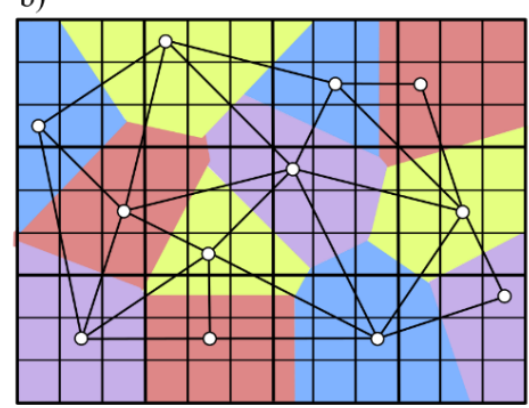

c)

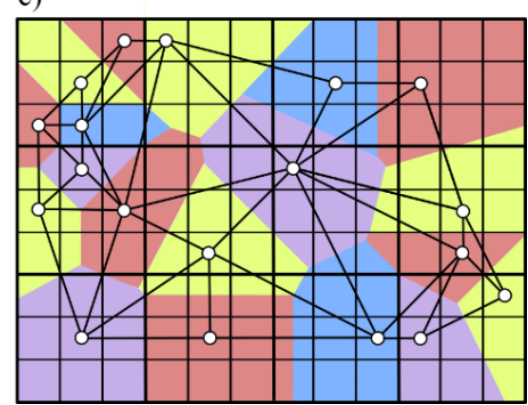

Figure 2. Adaptive sparse sampling. a) Scanning is initialized by randomly sampling a fixed percentage of pixels. b) Neighborhood relations are determined by computing a Voronoi diagram. c) The diagram is refined iteratively by placing additional samples on edges that fulfill some gradientbased subdivision criterion. 